\title{
BRIGHT AND DARK SOLITONS IN GENERALIZED KERR-LIKE MEDIA
}

\author{
J. JASIŃSKI \\ Faculty of Physics, Warsaw University of Technology \\ Koszykowa 75, 00-662 Warszawa
}

\begin{abstract}
In the paper the propagation of short pulses in nonlinear Kerr-like medium is considered. Pulses are described by the nonlinear Schrödinger equation in which the generalization of nonlinear dispersion term and the self-frequency shift are taken into account. The solution of the equation in the form of quadrature is derived. Both the phase and profile of the pulse intensity are obtained. The cases of propagating bright and dark solitons in saturable Kerr-like medium are analyzed. Two models of saturation are considered. The explicit analytical expressions describing the Kerr limit are reported. The relations between the medium and propagation parameters are thoroughly discussed.
\end{abstract}

PACS numbers: 42.65.Tg, 42.82.Et

\section{Introduction}

In nonlinear Kerr dielectric strong light pulses of special shape (solitons) propagate without changing its form due to the compensation of two effects - the linear group velocity dispersion (GVD) and nonlinear polarization of the medium (self-phase modulation, SPM) [1-3]. Propagation of these pulses is described by nonlinear Schrödinger equation (NLSE).

The strong electric field of soliton can cause many other effects in the Kerr dielectric. One of these effects - the third or higher order dispersion - is linear [4-6]; the others are nonlinear. In the paper two of such effects are considered nonlinear dispersion (ND) [6-9] and self-frequency shift (SFS) [10, 11]. The NLSE equation generalized by these (GNLSE) terms also possesses solutions in the form of solitons, but special requirements between parameters of the medium and soliton are needed in order to preserve the original profile from the pure Kerr dielectric $[6,12-16]$.

In the presence of strong soliton field the nonlinear permittivity of the medium can deviate from the simple Kerr dependence revealing saturation. The way we should modify it depends on model of saturation. By now the two-level model [17], the exponential model [18], "square two-level" [19], and polynomial 
models (qubic-quintic) [20-22] have been used most frequently. Since all these models give very similar results for small fields (far from saturation), application of any of them is the matter of convenience.

The nonlinear permittivity function determines the form of higher order nonlinear terms in GNLSE, so its modification affects these terms. For permittivity being arbitrary function of light intensity (describing non-Kerr but Kerr-like medium) we can easily realize such change with respect to ND and SFS terms. The obtained GNLSE equation is possible to solve for bright soliton case [23]. Its solution has a form of a quadrature. Solitons in saturable dielectrics described by this quadrature exist in much wider regime of parameters of medium and soliton itself compared to the Kerr case.

The above method can be modified to describe dark solitons as well. In the presented paper we expand this solution to comprise both bright and dark soliton cases and discuss the case of dark soliton, whose propagation in generalized Kerr-like medium has not been analyzed yet.

\section{The solution of GNLSE}

The nonlinear medium reacts to the travelling wave changing its dielectric permittivity $\varepsilon$ by $\varepsilon_{\mathrm{NL}}$. This response of the medium for Kerr-like dielectrics depends on light intensity $I=|\boldsymbol{E}|^{2}$ and for the pure Kerr case is simply proportional to intensity

$$
\varepsilon_{\mathrm{NL}}(I)=\alpha I \text {. }
$$

If the field intensity is sufficiently strong, the nonlinear part of permittivity deviates from the rule (1). The natural reason of this deviation is effect of saturation. The quantitative description of this effect depends on applied physical or mathematical model. Physically, the two-level model of saturation is the most justified [24]

$$
\varepsilon_{\mathrm{NL}}(I)=\frac{\alpha I}{1+I / I_{\mathrm{s}}},
$$

with $I_{\mathrm{S}}$ being the parameter of saturation (saturation intensity), but for mathematical convenience a few other functions expressing $\varepsilon_{\mathrm{NL}}$ can be assumed. Quite frequently we use the exponential function instead of (2)

$$
\varepsilon_{\mathrm{NL}}(I)=\frac{\alpha I_{\mathrm{s}}}{2}\left[1-\exp \left(-2 I / I_{\mathrm{s}}\right)\right]
$$

with $I_{\mathrm{s}}$ playing the same role as in (2) (the exponential model of saturation [18]) or apply the polynomial function (qubic-quintic model) [20]

$$
\varepsilon_{\mathrm{NL}}(I)=\alpha I-\alpha I^{2} / I_{\mathrm{s}} \text {. }
$$

Since both functions (2) and (3) have Taylor expansions up to second-order terms exactly in the form (4), therefore all above models should give the same results in the regime of small intensities $I \ll I_{\mathrm{s}}$.

Any of the functions (2)-(4) modifies the SPM term of nonlinear Schrödinger equation $[17,18,20-23]$. But modification of nonlinear permittivity of the medium should affect the other nonlinear effects being consequences of interaction between 
light and medium. Since the corrections to field distribution caused by these effects are small, we do not need to change the terms describing these effects if the fields are small. Nevertheless, the generalization of these terms can be performed without any trouble $[8,23]$. Neglecting the linear third-order dispersion effect and all processes in which energy is lost, we can write the GNLSE for the slowly varying envelope $U(z, t)$ of the transverse component of the electric field $E(z, t)$ in the frame traveling along the $z$-direction with the group velocity $v_{\mathrm{g}}=\mathrm{d} \omega / \mathrm{d} k$ of the packet $[23]$

$$
\begin{aligned}
\frac{\partial U}{\partial z} & +\frac{\mathrm{i} k_{2}}{2} \frac{\partial^{2} U}{\partial t^{2}}-\mathrm{i} \varepsilon_{\mathrm{NL}}\left(|U|^{2}\right) U+\frac{2}{\omega} \frac{\partial}{\partial t}\left[\varepsilon_{\mathrm{NL}}\left(|U|^{2}\right) U\right] \\
& +\tau U \frac{\partial}{\partial t} \varepsilon_{\mathrm{NL}}\left(|U|^{2}\right)=0 .
\end{aligned}
$$

In this equation $k_{2}$ determines the group velocity dispersion, $\omega$ is the carrier wave frequency and $\tau$ (real) is the retardation time. The light intensity is defined by the square of the absolute value of the slowly varying envelope: $I=|U|^{2}$.

Let us decompose (5) into parts determining the phase $\Phi(z, t)$ of the pulse and its intensity. The equation for the phase can be integrated twice giving [23]

$$
\begin{aligned}
& \Phi(z, t)=\frac{k_{2}}{2}\left(\frac{L}{T^{2}}-\Omega^{2}\right) z-\Omega T s \\
& +\frac{2 T}{\omega k_{2}} \int_{0}^{s}\left\{(\kappa+1) \varepsilon_{\mathrm{NL}}[I(s)]-\frac{\kappa F[I(s)]}{I(s)}\right\} \mathrm{d} s .
\end{aligned}
$$

In the above expression $L$ is one of the integration constant (the second one has been set to zero [7]), $T$ and $1 / \Omega$ are temporal and spatial widths of the pulse, $s$ is the normalized coordinate in the frame propagating with the maximum of the envelope

$$
s=\frac{t-k_{2} \Omega z}{T},
$$

$\kappa$ is combined nonlinear corrections parameter that characterizes both ND and SFS

$$
\kappa=1+\omega \tau
$$

while $F(I)$ is the auxiliary function defined by means of nonlinear permittivity $\varepsilon_{\mathrm{NL}}(I)$

$$
F(I)=\int_{0}^{I} \varepsilon_{\mathrm{NL}}(I) \mathrm{d} I .
$$

Note that fields that do not change their shape during propagation depend on one variable only: $I=I(s)$ in the frame connected with their own maximum.

The intensity function $I(s)$ satisfies the equation that follows from GNLSE after elimination of the phase function $\Phi(z, t)$. This equation is quite complicated and can be integrated only once. Nevertheless, the solution of the resulting ordinary first-order differential equation can be written as quadrature

$$
s(I)= \pm \int_{I}^{I_{0}} \frac{\mathrm{d} I}{\sqrt{\frac{16 \kappa^{2} G(I)}{q \alpha^{2} I_{\mathrm{K}}^{2}}+\frac{8 p I F(I)}{\alpha I_{\mathrm{K}}}-4 L I^{2}+4 J I}} .
$$


In the above integral $G(I)$ is another function built with the help of nonlinear permittivity $\varepsilon_{\mathrm{NL}}(I)$

$$
G(I)=\frac{\kappa^{2}-1}{\kappa^{2}} I \int_{0}^{I} \varepsilon_{\mathrm{NL}}^{2}(I) \mathrm{d} I-\left(\int_{0}^{I} \varepsilon_{\mathrm{NL}}(I) \mathrm{d} I\right)^{2},
$$

$q$ and $I_{\mathrm{K}}$ are the temporal width and Kerr intensity parameters

$$
\begin{aligned}
& q=(2 \Omega+\omega)^{2} T^{2}, \\
& I_{\mathrm{K}}=\frac{\omega}{(2 \Omega+\omega) T^{2}}\left|\frac{k_{2}}{\alpha}\right|,
\end{aligned}
$$

$p$ is the sign of the ratio of group velocity dispersion coefficient to the constant of the Kerr nonlinearity: $p=\operatorname{sgn}\left(k_{2} / \alpha\right)= \pm 1$ and $J$ is another constant of integration.

The quadrature (10) generalizes results reported in [23] for both cases of bright and dark solitons. The type of the field that propagates along the $z$ direction depends on $J, p$ and the maximum (or minimum) field intensity $I_{0}$, appearing in the upper limit of integration of (10). In the pure Kerr medium the bright soliton case is characterized by vanishing constant of integration $J$, positive maximum intensity $I_{0}$ and negative sign coefficient $p=-1$. On the other hand, dark solitons appear for positive $J$, vanishing minimum intensity $I_{0}$, and positive sign of $k_{2} / \alpha$.

\section{Solitons in saturable Kerr-like media}

The integral in quadrature (10) can be performed in both bright and dark soliton case for nonlinear permittivity function $\varepsilon_{\mathrm{NL}}(I)$ given by (1) and (4), that is for pure Kerr nonlinearity and for polynomial model of saturation. In the generalized Kerr (GK) case (that is for Kerr nonlinearity (1) and non-vanishing two last terms in (5)) we can solve the resulting equation with respect to $I$ obtaining the explicit function $I(s)$ describing profile of the pulse. Doing so for the bright soliton $(J=0$ and $p=-1)$ we arrive at the expression

$$
I(s)=\frac{(1+\sigma) I_{\max }}{\cosh ^{2} s+\sigma},
$$

in which the maximum value of intensity $I_{\max }=I_{0}$ and the broadening parameter $\sigma$ are defined by means of material and soliton parameters $\kappa, q$, and $I_{\mathrm{K}}$ as [23]

$$
\begin{aligned}
& \sigma=\frac{1}{2}\left[1-\frac{4\left(\kappa^{2}-4\right)}{3 q}\right]^{-1 / 2}-\frac{1}{2} \\
& I_{\max }=\frac{1+2 \sigma}{1+\sigma} I_{\mathrm{K}} .
\end{aligned}
$$

The similar integration performed for the dark soliton $\left(I_{0}=0\right.$ and $\left.J>0\right)$ gives its profile described by analogous expression

$$
I(s)=\frac{(1-\sigma) I_{\infty} \sinh ^{2} s}{\cosh ^{2} s-\sigma \sinh ^{2} s},
$$

but meaning of the parameter $I_{\infty}$, playing the role of the soliton height is different. Since $I(0)=0$, we should take the background intensity $I_{\infty}=\lim _{s \rightarrow \pm \infty} I(s)$ as 
the measure of the height. This height can be determined by broadening parameter $\sigma$ and the Kerr intensity $I_{\mathrm{K}}$ in a different way than that given in formula (16)

$$
I_{\infty}=p I_{\mathrm{K}}(1-3 \sigma) \text {. }
$$

The broadening parameter itself depends on nonlinear corrections $\kappa$, temporal width parameters $q$ and sign coefficient $p$ in a quite complicated manner

$$
\sigma=\frac{\left|1+\gamma \mp p \sqrt{\gamma^{2}+\gamma}\right|}{3},
$$

where $\gamma$ is defined by $\kappa$ and $q$ as

$$
\gamma=\frac{q}{2\left(\kappa^{2}-4\right)}
$$

Two signs + and - (two branches of solutions) are possible only in the case $p=+1$ and $\kappa^{2}<4$. In all other cases only upper sign $(-)$ should be taken. The second branch of the solution disappears in the pure Kerr limit.

The analytical solution (17)-(20) exists for both type of sign coefficient $p= \pm 1$. It contrasts with the pure Kerr medium case $\left(\varepsilon_{\mathrm{NL}}(I)\right.$ given by $(1)$ and vanishing two last terms in GNLSE), in which dark solitons appear only for $p=+1$. But since the physical meaning have only positive values of intensity $I(s)$ and $I_{\infty}$, certain combinations of material and soliton parameters cannot be taken into account.

The integration given by $(10)$ is also possible to perform for media with saturable nonlinear permittivity described by polynomial model (4). But the resulting function $s(I)$ for both bright and dark soliton cases is expressed by a combination of elliptic integrals of the first and third kind. The explicit form of these integrals for bright solitons has been published in [23]. Dark solitons are described by more complicated expressions and we shall not report them. But still they enable to draw the profiles of the solitons.

Nevertheless, in the two-level and exponential models of saturation the functions $F(I)$ and $G(I)$ defined by $(9)$ and (11) can be expressed by analytical formulas, the integration in quadrature (10) must be performed numerically. In Fig. 1 we compare the resulting profiles for the GK case (without saturation) and all considered models of saturation for the dark solitons (the case of bright soliton was widely analyzed in [23], therefore further discussion will concern the dark solitons case). In order to increase the differences between models we took large height of the soliton compared to intensity of saturation $I_{\mathrm{K}} / I_{\mathrm{S}}=0.4$. We can see that for any model of saturation the propagating solitons have very similar profiles and height significantly greater than the height in GK medium. This height $I_{\infty}$ in the exponential model is slightly greater than $I_{\infty}$ in the two-level saturation and this property takes place for any choice of parameters. The approximate qubic-quintic model gives solitons of height lying between two other types of saturation, but for another parameters we can obtain another relation.

The influence of saturation on the background intensity (height of dark soliton) $I_{\infty}$ can be observed in Fig. 2. If the height of soliton in the pure Kerr case $I_{\mathrm{K}}$ is at least one order smaller than intensity of saturation $I_{\mathrm{s}}$, we cannot practically 


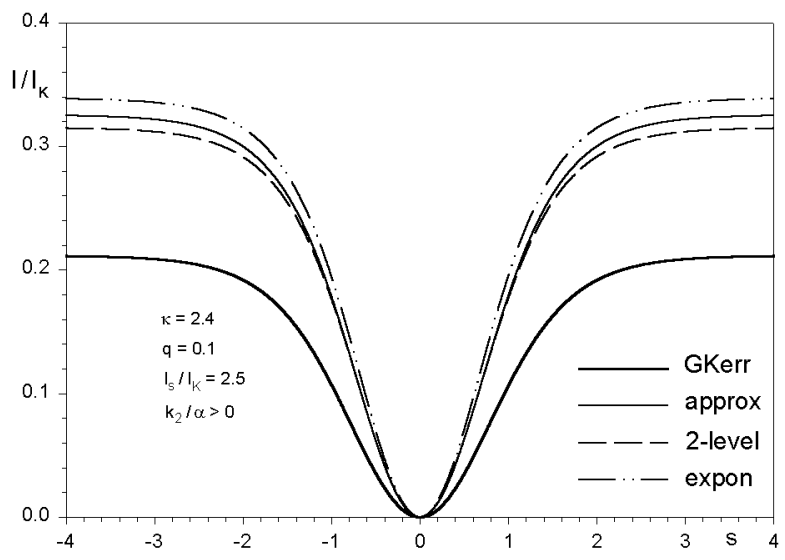

Fig. 1. Profiles of the dark solitons in generalized Kerr (GKerr) medium (without saturation) and three models of saturation: approximate (approx), two-level, and exponential (expon).

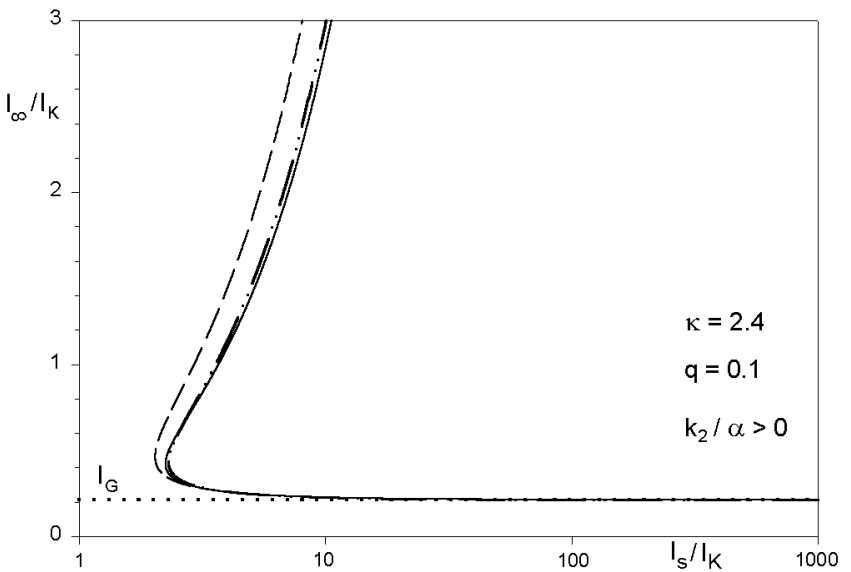

Fig. 2. The height of the dark solitons (background intensity) versus intensity of saturation in three models of saturation compared to the height in the generalized Kerr medium ( $I_{\mathrm{G}}$, dotted line). The designation of lines is the same as in Fig. 1.

distinguish any saturable medium from the pure Kerr dielectric, nevertheless we consider ND and SFS terms or not. On the contrary to the pure Kerr case, the height of soliton cannot be too large - the maximum value $I_{\mathrm{K}}$ is about one third $I_{\mathrm{s}}$ (it depends on model of saturation and other parameters). For larger solitons (or smaller level of saturation) we have two branches of solutions (for bright soliton case we have only one branch). The second branch gives solitons much higher than for the pure Kerr case. For this branch the exponential model gives smaller background intensity $I_{\infty}$. 
The relation between soliton height and $\kappa$ is more complex. For quite small level of saturation $I_{\mathrm{s}} / I_{\mathrm{K}}=5$ we have two distinct branches of solutions for GK case and two distinct branches for approximate model (Fig. 3). The lower branches exist for any $\kappa$, but the regime of upper ones is narrower. Solitons in the GK case appear for small $\kappa$, while solitons in the approximate model for large $\kappa$. For the two other models of saturation we have also two branches of solutions, but both of them exist for large $\kappa$ and extend each other. For larger level of saturation the upper branch in the GK case disappears, the branches in 2-level and exponential models change only slightly and the case of approximate model becomes similar to other saturable cases.

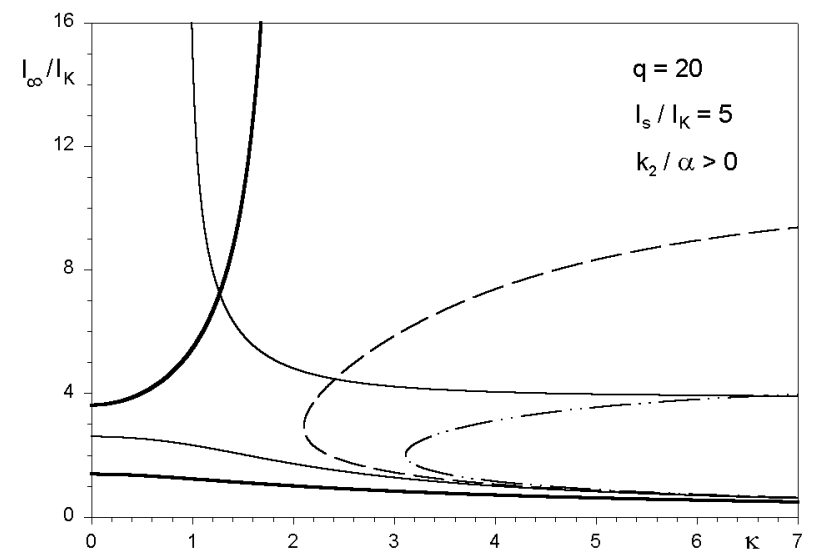

Fig. 3. The heights of the dark solitons as functions of nonlinear corrections. Lines are designated as in Fig. 1.

The height of soliton as function of temporal width parameter $q$ depends on the sign $p$ of $k_{2} / \alpha$, the nonlinear correction parameter $\kappa$, and the model of saturation. The whole classification obeys a lot of cases and is quite difficult to carry out. In Fig. 4 we show four different types of relations $I_{\infty}(q)$ for a few sets of other parameters. One of them (the upper left diagram) is obtained for $p=-1$ and shows functions $I_{\infty}(q)$ differing very much between saturable and GK case. On the other hand, for $p=+1$ the GK case is much more similar to the other saturable cases (lower left plots), however the regime of the second branch in the GK case is rather narrow. Both cases are obtained for quite large levels of saturation and are typical of this case. But the slightly lower value of $I_{\mathrm{s}}$ gives completely different relation $I_{\infty}(q)$. As we can see, the 2 -level model of saturation enables existence of solitons in the regime of $q$ forbidden for them in the other models (the upper right plots). The curves in lower right part of Fig. 4 are plotted for the smallest value of saturation. Comparing with the previous diagram we observe that the line characterizing solitons with large temporal width $q$ in the exponential saturable medium disappeared, while lines corresponding to approximate model of saturation joined together and split into two another parts — describing solitons with small 

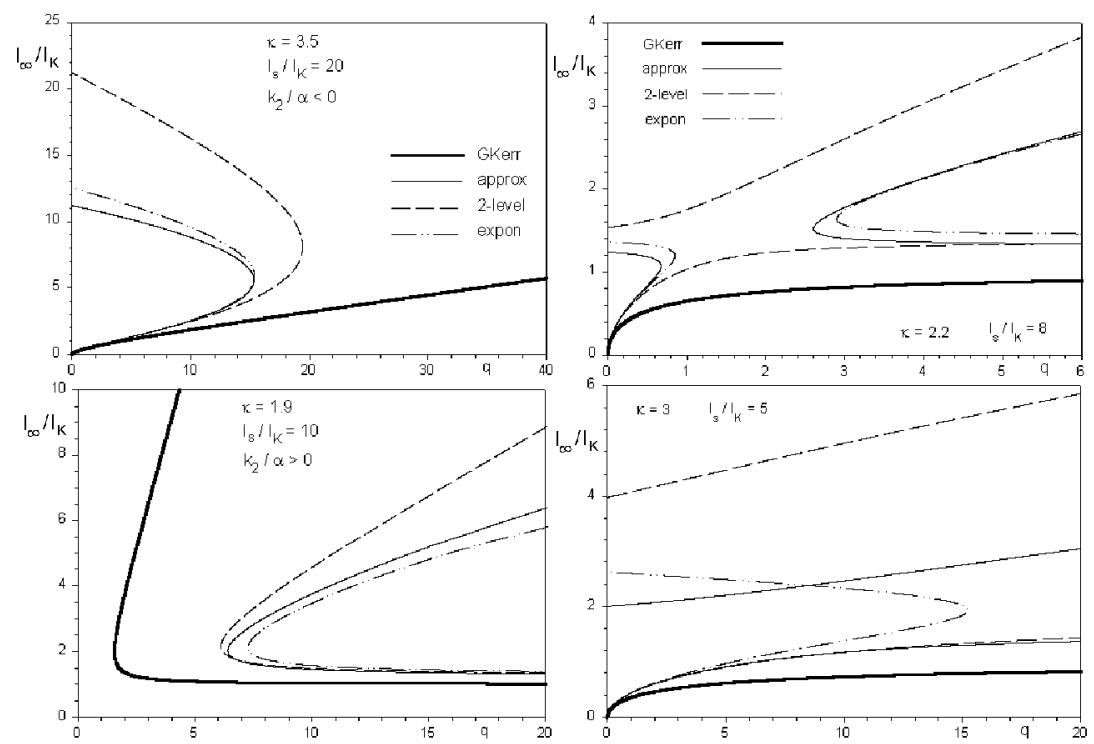

Fig. 4. The heights of the dark solitons as functions of temporal width. Upper left diagram made for $k_{2} / \alpha<0$, three others for $k_{2} / \alpha>0$. The lines are designated as in Fig. 1.

and large height. We have two possibilities of $I_{\infty}$ for any $q$ for two-level model of saturation.

However, there are many other configurations of lines $I_{\infty}(q)$, we can observe several common features. First, in any of diagrams we have a certain regime in which all saturable solutions are similar and distinctly differ from solution in GK medium. Second, there are regimes of parameters in which there are no saturable solutions and these regimes for different types of saturation are different. Generally, the widest regime of parameters is possible in the two-level model saturation. Third, all saturable solutions, if exist, are double. It contrasts with the GK case in which we have a single solution for most possible parameters. Four, the highest values of $I_{\infty}$ in the upper branch and the least values in the lower branch (however, sometimes the least height is obtained in approximate case) result in two-level model of saturation.

Generally, we can say that the presence of saturation expands the set of solutions of GNLSE very significantly.

\section{Summary and conclusions}

The GNLSE (5) possesses solutions in the form of both bright and dark solitons. The intensity profiles of these solitons are given by quadrature (10). The integration in the quadrature can be performed analytically for nonlinear permittivity function describing Kerr medium and Kerr-like saturable qubic-quintic medium, but only in the first case we obtain simple formulae (14), (17). The other models of saturation require numerical calculations. 
If the height of soliton is small comparing to the intensity of saturation, all saturable models give similar results (Fig. 1). But in saturable generalized medium, solitons of larger intensity can differ very much from solitons in pure Kerr medium. The differences are particularly evident for dark solitons (Figs. 2-4). Such solitons appear in the medium with $k_{2} / \alpha<0$, where dark Kerr solitons cannot exist. But certain regimes of parameters are forbidden for saturable cases, however in the Kerr medium, solitons of such parameters do exist. For any choice of parameters saturable solutions given by quadrature (10), if exist, are double. Moreover, the model of saturation influences very significantly on the behavior of solution (Fig. 4). The widest regime of possible solutions characterizes the two-level model of saturation.

\section{References}

[1] G.P. Agrawal, Nonlinear Fiber Optics, Academic Press, San Diego 1995, p. 28.

[2] V.B. Zakharov, A.B. Shabath, Sov. Phys. JETP 34, 62 (1972).

[3] G.P. Agrawal, in: Contemporary Nonlinear Optics, Eds. G.P. Agrawal, R.W. Boyd, Academic Press, San Diego 1995, p. 41.

[4] J.N. Elgin, Opt. Lett. 17, 1409 (1992).

[5] M. Piché, J.-F. Cormier, X. Zhu, Opt. Lett. 21, 845 (1996).

[6] G. Dong, Z. Liu, Opt. Commun. 128, 8 (1996).

[7] D. Anderson, M. Lisak, Phys. Rev. A 27, 1393 (1983).

[8] R.H. Enns, S.S. Rangnekar, Phys. Rev. A 43, 4047 (1991).

[9] J.R. de Oliveira, M.A. de Moura, J.M. Hickman, A.S.L. Gomes, J. Opt. Soc. Am. B 9, 2025 (1991).

[10] J.P. Gordon, Opt. Lett. 11, 662 (1986).

[11] Y.S. Kivshar, B.A. Malomed, Opt. Lett. 18, 485 (1993).

[12] G.P. Agrawal, C. Headley, Phys. Rev. A 46, 1573 (1992).

[13] K. Hizanidis, D.J. Frantzeskakis, C. Polymilis, J. Phys. A 29, 7687 (1996).

[14] M. Gedalin, T.C. Scott, Y.B. Band, Phys. Rev. Lett. 78, 448 (1997).

[15] S. Liu, X. Liu, Phys. Lett. A 225, 67 (1997).

[16] S.L. Palacios, J.M. Fernández-Díaz, A. Guinea, J. Mod. Opt. 47, 711 (2000).

[17] S. Gatz, J. Herrmann, J. Opt. Soc. Am. B 8, 2296 (1991).

[18] U. Langbein, F. Lederer, T. Peschel, H.E. Ponath, Opt. Lett. 10, 571 (1985).

[19] W. Królikowski, X. Yang, B. Luther-Davies, J. Breslin, Opt. Commun. 105, 219 (1994).

[20] F.G. Bass, V.V. Konotop, S.A. Puzenko, Phys. Rev. A 46, 4185 (1992).

[21] H.W. Schürmann, Phys. Rev. E 54, 4312 (1996).

[22] W. Chao, Opt. Commun. 175, 239 (2000).

[23] J. Jasiński, Opt. Commun. 172, 325 (1999).

[24] R.W. Boyd, Nonlinear Optics, Academic Press, Boston 1992, p. 191. 